\title{
INTRAOPERATIVE SENTINEL LYMPH NODE MAPPING IN LAPAROSCOPIC OPERATIONS FOR COLORECTAL CANCER
}

\author{
Venelin Georgiev, Panayot Kurtev, Lachezar Dzhongov, Lyubomir Vartanyanov, \\ Elka Angelova, Lilyan Lukov, Eva Dimanova, Kalin Enikov, Yordan Simeonov, \\ Vladislav Yankov \\ Clinic for Abdominal Surgery, University Specialized Hospital for Active Treatment \\ in Oncology, Sofia, Bulgaria
}

\begin{abstract}
INTRODUCTION: Laparoscopic resections in colorectal cancer gain an increasing popularity. Sentinel lymph node mapping in colorectal cancer and its advantages in daily practice are still subject of discussion. In laparoscopic operations, the procedure has same technical difficulties, although provides definite advantages in staging and estimating the extent of lymph dissection.

MATERIALS AND METHODS: We present techniques for intraoperative sentinel lymph nodes mapping in colorectal cancer and their detection with surgical gamma probe in laparoscopic resection, which are used in our clinic, and the initial results from them.

RESULTS: The initial results show that sentinel lymph nodes mapping with radionuclides and their detection with surgical gamma probe are technically feasible in laparoscopic resections for colorectal cancer. The method has additional possibilities for the surgeon for estimating of the lymph drainage and precise staging in laparoscopic operation.

CONCLUSION: Nevertheless the contradictory opinions for application of sentinel lymph nodes mapping in colorectal cancer, the method provides certain advantages in improving staging. In laparoscopic operations, although having some difficulties, it could be used, as it offers additional facilitation for the surgeon in estimating the lymph drainage in condition of decreased tactile sensation. Scr Sci Med 2017; 49(3):19-21

Keywords: colorectal cancer, laparoscopic operations, sentinel lymph nodes, radionuclides

Address for correspondence:

Venelin Georgiev

Clinic for Abdominal Surgery,

University Specialized Hospital for Active Treatment in

Oncology,

"Plovdivsko Pole" str. 6

Sofia, Bulgaria

e-mail:venelin_georgiev@hotmail.com

Received: July 31, 2017

Accepted: September 15, 2017

\section{INTRODUCTION}

Colorectal cancer is leading cause of death in oncological diseases. Metastases in regional lymph nodes are one of the most important prognostic factors in malignant tumors including the cancer of colon and rectum. Patients with lymph node involvement have lesser survival and need adjuvant chemotherapy. It is established that $20-40 \%$ of patients with tumors infiltrating the muscular layer of the wall, who do not have detected lymph metastases, eventu-
\end{abstract}


ally die from the oncological disease. One of the reasons for this is the failure to detect existing metastases in regional lymph nodes (1). The substantial number of lymph nodes sampled from the specimen after resection makes their exact examination difficult. Sentinel lymph node biopsy enables a more exact determination of patient's lymph status and a more precise staging, because it allows focused examinationc such as immunohistochemistry, serial sections, etc. due to the smaller lymph node number.

J. J. Joosten et al. first used the technique for sentinel lymph node mapping with dyes in colorectal cancer (2). Very soon after their publication, D. A. Wiese et al. (3) and S. Saha et al. (4) first described a successful technique for sentinel node detection using the blue dye isossulfan blue (lymphazurin). Lymphatic mapping in laparoscopic resection was first published by T. F. Wood et al. in 2001.

Y. Kitagawa first reported the application of radionuclides for sentinel lymph node mapping in colorectal cancer (6). S. Saha et al. (7) comparatively analyzed these two methods for sentinel lymph node mapping - by means of dyes and of radionuclides.

Laparoscopic resections in colorectal cancer are now routinely done for colorectal cancer. These authors presented adequate oncological results without significant increased risk for the patient in terms of postoperative morbidity and lethality. In the same time, laparoscopic operation has some advantages in comparison to the open surgery: lesser blood loss, less surgical wound complications, better esthetic outcome, reduced postoperative pain, lesser frequency of postoperative hernias, smaller hospital stay, etc. One of the main disadvantages of laparoscopic resections is the lack of direct tactile sensation in the surgeon. This is very important especially in obese patients. The technique of sentinel lymph node mapping offers additional possibilities for more precise determination of the lymph drainage in these cases.

\section{MATERIALS AND METHODS}

On the base of the methods described in the scientific literature we have developed one-day protocol for sentinel lymph node mapping in colorectal cancer using radionuclides. Its main advantage is that marking is done during the operation and preoperative endoscopic injecting of the marker is not necessary. Laparoscopic resections are associated with additional difficulties in the procedure of sentinel lymph node mapping connected to more difficult localization of the tumor and injecting of the marker and the necessity of specific devises for laparoscopic detection. There are a few publications in the literature for use of radionuclides in laparoscopic resections for sentinel lymph node mapping in colorectal cancer. The use of dyes is the preferred technique. The use of radionuclides has significant advantages especially in detecting of lymph nodes covered with a lot of tissue, for example in obese patients.

In our hospital, we have used the following method for intraoperative sentinel lymph node mapping in laparoscopic operations for colorectal cancer: In case of distal rectal cancer $1 \mathrm{ml} 1 \mathrm{mCi} 99 \mathrm{~m}$ Tc nanocolloid is injected on four places submucosally around the tumor in the beginning of the intervention. In all other cases of colorectal cancer, we use spinal needle for injection through abdominal wall under visual control by laparoscopic camera in four places around the tumor subserosally. Thirty minutes later we use laparoscopic probe of surgical gamma-probe Europrobe II through a port $10 \mathrm{~mm}$ to detect the distribution of radioactivity. After extracting the specimen all lymph nodes are dissected and the level of radioactivity in them is detected.

\section{RESULTS}

The initial results show that sentinel lymph nodes mapping with radionuclides and their detection with surgical gamma probe are technically feasible in laparoscopic resections for colorectal cancer. The method has additional possibilities for the surgeon for estimating of the lymph drainage and precise staging in laparoscopic operation.

\section{CONCLUSION}

The method of sentinel lymph node mapping in colorectal cancer is still a subject to discussion, but the studies till now show its advantages for improved staging and intraoperative determination of lymph drainage. Nevertheless, some technical difficulties this method can be used in laparoscopic resection, which gain more popularity in everyday practice. In laparoscopic resections advantages of sentinel lymph node mapping are bigger due to the lack of direct tactile sensation in the surgeon. The use of radionuclides as a marker has advantages to dyes, especially 
in obese patients and deep located lymph nodes with difficult visual identification. The main disadvantage is the necessity of special equipment.

\section{REFERENCES}

1. Dubé S, Heyen F, Jenicek M. Adjuvant chemotherapy in colorectal carcinoma: results of a meta-analysis. Dis Colon Rectum. 1997;40(1):35-41.

2. Joosten JJ, Strobbe LJ, Wauters CA, Pruszczynski $\mathrm{M}$, Wobbes T, Ruers TJ. Intraoperative lymphatic mapping and the sentinel node concept in colorectal carcinoma. Br J Surg. 1999;86(4):482-6. doi: 10.1046/j.1365-2168.1999.01051.x.

3. Wiese DA, Saha S, Badin J. Sentinel lymph node mapping in staging of colorectal carcinoma. Am J Clin Pathol. 1999;112:542.

4. Saha S, Wiese D, Badin J, Beutler T, Nora D, Ganatra BK, et al. Technical details of sentinel lymph node mapping in colorectal cancer and its impact on staging. Ann Surg Oncol. 2000;7(2):120-4.

5. Wood TF, Saha S, Morton DL, Tsioulias GJ, Rangel D, Hutchinson W Jr, et al. Validation of lymphatic mapping in colorectal cancer: in vivo, ex vivo, and laparoscopic techniques. Ann Surg Oncol. 2001;8(2):150-7.

6. Kitagawa $Y$, Watanabe $M$, Hasegawa $H$, Yamamoto S, Fujii H, Yamamoto K, et al. Sentinel node mapping for colorectal cancer with radioactive tracer. Dis Colon Rectum. 2002;45(11):1476-80.

7. Saha S, Dan AG, Berman B, Wiese D, Schochet E, Barber K, et al. Lymphazurin $1 \%$ versus $99 \mathrm{mTc}$ sulfur colloid for lymphatic mapping in colorectal tumors: a comparative analysis. Ann Surg Oncol. 2004;11(1):21-6. 